\title{
Ansiedad por Covid - 19 y salud mental en estudiantes universitarios
}

\section{Coronavirus anxiety and the mental health in university students}

\author{
Andrea Vivanco-Vidal ${ }^{1}$ \\ Cencosud del Grupo Scotiabank \\ Daniela Saroli-Araníbar ${ }^{2}$ \\ Markham College \\ Tomás Caycho-Rodríguez ${ }^{3}$, Carlos Carbajal-León ${ }^{4}$ \\ Universidad Privada del Norte \\ Martín Noé-Grijalva ${ }^{5}$ \\ Universidad César Vallejo
}

Recibido: 13 - $09-20$

Aceptado: $16-11-20$

Publicado: $21-12-20$

\section{Resumen}

El objetivo de la presente investigación fue determinar la relación entre ansiedad por Covid - 19 y salud mental en 356 estudiantes universitarios (227 mujeres y 129 hombres, $\mathrm{M}_{\text {edad }}=$ 22.36 años, $\mathrm{DE}=2.46$ ). Asimismo, se comparó la ansiedad por Covid - 19 y salud mental entre algunas variables sociodemográficas. Se aplicó la versión en español de la Coronavirus Anxiety Scale (CAS) y el Mental Health Inventory-5 (MHI). Los resultados muestran que una mayor ansiedad por COVID - 19 se relaciona con una disminución de la salud mental $(\rho=$ $-.67, \mathrm{p}<.01$ ). Asimismo, respecto a las comparaciones realizadas se evidencian diferencias estadísticamente significativas en función a las variables sociodemográficas previamente

\footnotetext{
1 Bachiller en Psicología por la Universidad Peruana de Ciencias Aplicadas, Lima, Perú. Labora en Cencosud del Grupo Scotiabank.

Autor para correspondencia: U201414146@upc.edu.pe ORCID: http://orcid.org/0000-0003-1546-7167

2 Bachiller en Psicología por la Universidad Peruana de Ciencias Aplicadas, Lima, Perú. Labora en Markham College. E-mail: U201510766@upc.edu.pe ORCID: http://orcid.org/0000-0001-6655-0214

3 Docente de la Universidad Privada del Norte, Lima, Perú.

E-mail: tomas.caycho@upn.pe ORCID: http://orcid.org/0000-0002-5349-7570

4 Docente en la Universidad Privada del Norte, Lima, Perú.

E-mail: alberto.carbajal@upn.pe ORCID: http://orcid.org/0000-0001-7439-2332

5 Docente en la Universidad César Vallejo. Trujillo, Perú.

E-mail: hnoe@ucv.edu.pe ORCID: http://orcid.org/0000-0003-2224-8528
}

(C) Los autores. Este artículo es publicado por la Revista de Investigación en Psicología de la Facultad de Psicología, Universidad Nacional Mayor de San Marcos. Este es un artículo de acceso abierto, distribuido bajo los términos de la licencia Creative Commons Atribucion - No Comercia_Compartir Igual 4.0 Internacional. (http://creativecommons.org/licenses/by-nc-sa/4.0/) que permite el uso no comercial, distribución y reproducción en cualquier medio, siempre que la obra original sea debidamente citada. 
mencionadas. El estudio confirma que mayor ansiedad por COVID-19 se relaciona con un empeoramiento de la salud mental en una muestra de estudiantes universitarios peruanos.

Palabras clave: Ansiedad; Salud mental; Universitarios, COVID - 19.

\begin{abstract}
The objective of the present study is to determine the relationship between anxiety by Covid - 19 and mental health in 356 university students ( 227 women and 129 men, average age $=$ 22.36 years, Standard Deviation $=2.46$ ). It also has as specific objectives to compare the anxiety by Covid - 19 and mental health according to sociodemographic variables. To whom it was applied the Coronavirus Anxiety Scale in Spanish and the Mental Health Inventory-5 (MHI). The study confirms that there is a statistically significant correlation between anxiety by Covid -19 and mental health $(\rho=-.67, p<.01)$. Also, regarding the comparisons made statistically significant differences are evidenced according to the variables sociodemographic previously mentioned. The study confirms that the more anxiety by Covid - 19 the lower mental health in a sample of Peruvians university students.
\end{abstract}

Keywords: Anxiety; Mental health; University students; COVID - 19.

El mundo actual exige a las personas el manejo apropiado de un conjunto de capacidades, habilidades y herramientas personales que les permitan adaptarse a las necesidades de la sociedad actual; mostrando autonomía, capacidad de constituir relaciones interpersonales positivas y el establecimiento de metas que permita su crecimiento en todo ámbito en el proceso de su autorrealización.

El nuevo coronavirus 2 del síndrome respiratorio agudo severo (SARSCoV-2) denominado enfermedad de 2019-nCoV o COVID - 19 surgió en Wuhan (China), en diciembre del 2019 (Lai, Shih, Ko, Tang, \& Hsueh, 2020), ocasionando rápidamente un brote de neumonía infecciosa aguda en diversos países (Bao, Sun, Meng, Shi, \& Lu, 2020). Por esa razón, en marzo del presente año la Organización Mundial de la Salud (OMS, 2020), basándose en el impacto del virus, la reconoció como pandemia. A la fecha, se han confirmado alrededor de 30 millones de casos de COVID - 19 y aproximadamente 950 mil muertes a nivel mundial (Johns Hopkins University \& Medicine, 2020). Asimismo, el Perú ocupa el quinto puesto en el mundo y el segundo en Latinoamérica en relación a los casos confirmados (Johns Hopkins University \& Medicine, 2020). Esta situación ha generado que se establezcan políticas gubernamentales de salud pública, orientadas primordialmente al distanciamiento social, para disminuir el riesgo de contagio (Jaramillo-Valverde \& Marquina, 2020), siendo la cuarentena obligatoria, una de las medidas con mayor repercusión, tanto positiva como negativa (Sanchez-Duque, Arce-Villalobos, \& Rodríguez-Morales, 2020).

En el caso de la salud mental, diversas investigaciones demuestran que durante la fase inicial de la pandemia aparecieron síntomas de ansiedad, depresión, pánico, angustia, miedo y reacción al estrés en la población general (Cao et al., 2020; Huarcaya-Victoria, 2020; Urzúa, Vera-Villarroel, Caqueo Urízar, \& 
Polanco-Carrasco, 2020; Wang \& Zhao, 2020). Estudios de epidemias y pandemias anteriores demuestran que la ansiedad, o la falta de ella, influye en el comportamiento (Taylor, 2019). Al respecto, las personas con ansiedad elevada son más propensas a participar de manera disruptiva, comprar excesivos materiales de higiene y se dirigirse de manera innecesaria a hospitales y clínicas por sentir dolencias insignificantes que interpretan como graves (Asmundson \& Taylor, 2020a; Asmundson \& Taylor, 2020b). Asimismo, bajos niveles de ansiedad, pueden ser nocivos y hacer creer a las personas que no pueden contagiarse y con ello, incumplir las normas de prevención (Asmundson \& Taylor, 2020b). En ese sentido, la presencia de mayores niveles de ansiedad en el contexto de la pandemia de COVID-19 se manifiesta debido a que los individuos pueden tener percepciones negativas de su salud, lo que aumenta su ansiedad, afecta su capacidad para tomar decisiones y comportarse de manera racional (Asmundson \& Taylor, 2020b).

Por otro lado, Shigemura, Ursano, Morganstein, Kurosawa, y Benedek (2020) refieren que las principales reacciones a nivel emocional en la población se relacionan al excesivo miedo e incertidumbre. De la misma manera, pueden presentarse conductas y estados que den inicio a problemas en la salud mental, como respuestas de angustia, comportamientos nocivos como mayor uso de alcohol y tabaco, estrés postraumático, ansiedad, depresión, y somatizaciones propias del miedo al contagio (Wang et al., 2020; Urzúa et al., 2020). Del mismo modo, Li, Wang, Xue, Zhao y Zhu (2020) encontraron que las emociones como la depresión, la ansiedad y la indignación aumentaron, al mismo tiempo que disminuyeron las emociones de felicidad y satisfacción con la vida.

Un sector fuertemente afectado por la pandemia de COVID - 19 es el educativo, donde el aprendizaje de los estudiantes se ha visto afectado por los obstáculos y las medidas impuestas por los gobiernos, que en su mayoría han suspendido las actividades presenciales de las instituciones educativas para prevenir la propagación de la enfermedad. Por esa razón, más del $60 \%$ de la población estudiantil del mundo se ha visto afectada, sobre todo las comunidades más vulnerables que pueden presentar dificultades para continuar sus estudios a través del aprendizaje remoto. Es el caso del Perú, en donde alrededor de 1.895.907 universitarios, entre hombres y mujeres, han sido perjudicados con respecto a sus estudios (UNESCO, 2020a). En ese sentido, la restricción del movimiento, la interrupción de rutinas, la disminución de la interacción social, la falta de métodos de aprendizaje tradicionales, así como las nuevas exigencias académicas, tecnológicas y psicológicas han generado un aumento de presión, estrés y ansiedad para los jóvenes (UNESCO, 2020b; Sanz, Sáinz \& Capilla, 2020).

$\mathrm{Al}$ respecto, la vida universitaria, representa una etapa en donde gran parte de los estudiantes son adolescentes o adultos jóvenes, con nuevas responsabilidades 
sociales y mayores presiones psicosociales, lo que puede suponer una mayor vulnerabilidad frente a trastornos psicosociales como el síndrome ansioso (CardonaArias, Pérez-Restrepo, Rivera-Ocampo, \& Gómez-Martínez, 2015). Diversos autores refieren que la ansiedad es un estado o condición emocional transitoria que se caracteriza por un sentimiento de tensión, aprensión difusa, un incremento de la actividad del sistema nervioso autónomo y desagradable, acompañado de síntomas como taquicardia, opresión torácica, malestar epigástrico e inquietud. Sin embargo, también puede ser una condición clínica normal, cuando es una señal de alerta que advierte una situación amenazante y permite al individuo tomar medidas para afrontarlo (Kaplan, Sadock, \& Sadock, 2009; Spielberger, Gorsuch, Lushene, Vagg, \& Jacobs, 1983).

Estudios realizados en China revelan que la salud mental de los jóvenes de nivel universitario se ve afectada de manera significativa al enfrentarse a la pandemia de la COVID-19. Los principales estresores relacionados con la COVID-19 son económicos, efectos sobre la vida cotidiana y retrasos académicos, que se asocian de manera positiva con los síntomas de ansiedad en estudiantes universitarios chinos durante la pandemia (Cao et al., 2020). También, los hallazgos recogidos en estudiantes universitarios en China indicaron que el $0.9 \%$ de los encuestados experimentaron ansiedad severa, 2.7\% ansiedad moderada y $21.3 \%$ ansiedad leve. Además, vivir en áreas urbanas $(\mathrm{OR}=0.810, \mathrm{IC} 95 \%=$ $0.709-0.925)$, estabilidad de ingresos familiares $(\mathrm{OR}=0.726$, IC $95 \%=0.645$ - 0.817) y vivir con los padres (OR $=0.752$, IC $95 \%=0.596-0.950)$ fueron factores protectores contra la ansiedad; mientras que, contar con familia o contactos infectados con la COVID-19 fue un factor de riesgo para incrementar la ansiedad de los estudiantes universitarios $(\mathrm{OR}=3.007$, IC 95\% $=2.377-3.804$; Cao et al., 2020).

Por otro lado, en América, el Perú realizó un estudio con universitarios de Lima se encontró que los participantes demostraron alta reactividad ante las variables ira y miedo con respecto al COVID - 19. Sin embargo, los ítems asociados con la dimensión de ansiedad mostraron un resultado promedio menor con relación al resto de los factores (Rodríguez, Aliaga, Peña-Calera, \& Quintana, 2020). Asimismo, un estudio en Puerto Rico, evidenció que los principales estresores en estudiantes universitarios durante la pandemia por COVID - 19, fueron la acumulación de tareas, la inadecuada ejecución de cursos virtuales, ausencia de asesoramiento, falta de comprensión de sus profesores, entre otros (Rosario-Rodríguez, González-Rivera, Cruz-Santos, \& Rodríguez-Ríos, 2020).

En alusión a lo mencionado, se puede evidenciar que los problemas de salud mental de las personas afectadas por la pandemia de la COVID-19 no han sido abordados de manera suficiente en el Perú, mucho menos en el ámbito educativo 
universitario. En este sentido, y a pesar de las evidencias mencionadas, no existe un estudio que relacione la ansiedad por Covid-19 y salud mental en estudiantes universitarios peruanos. Por esa razón, la presente investigación tiene como objetivo principal determinar la relación entre ambas variables. Asimismo, tiene como objetivos específicos: Comparar la ansiedad por Covid-19 y salud mental en función a la edad, sexo, convivencia familiar, contexto laboral, diagnóstico de la enfermedad en familiares, amigos y de ellos mismos, diagnósticos y tratamientos contra la sintomatología ansiosa, así como el tiempo que estaban pensando, escuchando o viendo en las noticias información sobre la COVID-19.

\section{MÉTODO}

\section{Diseño}

El presente estudio fue transversal y comparativo, debido a que los datos se recogieron en un solo momento del tiempo; además se compararon las variables salud mental y ansiedad por el COVID-19 entre diferentes variables sociodemográficas (Ato, López-García, \& Benavente, 2013)

\section{Participantes}

El presente estudio, se realizó en estudiantes de universidades públicas y privadas de las ciudades de Trujillo y Chimbote, cuyas edades oscilaron entre los 18 y 34 años, y que fueron seleccionados a través de un muestreo no probabilístico. El número de participantes se determinó mediante el software de Soper (2020), con un tamaño del efecto $d$ Cohen $=0.05$, un poder estadístico de 0.80 y una probabilidad de 0.05. Así, el tamaño mínimo esperado fue de 128 participantes. Buscando una mejor representatividad, en el presente estudio se optó por seleccionar más del doble de participantes sugeridos.

Participaron un total de 356 estudiantes de nivel superior, de los cuales el $63.8 \%(\mathrm{~N}=227)$ eran del sexo femenino y el 36.2\% ( $\mathrm{N}=129)$ fueron del sexo masculino, con una edad media de 22.36 años ( $\mathrm{DE}=2.46$ ). El 93.5\% de los participantes fueron solteros, el $71.6 \%$ desempleados y el $81.1 \%$ vivían con ambos padres o alguno de ellos. Cuatro personas fueron diagnosticadas con la COVID-19, sin embargo, el 100\% manifestó tener algún familiar diagnosticado con la COVID 19. Del mismo modo, la gran cantidad indicó no tener conocidos con el diagnostico con COVID-19 (76.1\%). En las dos últimas semanas, el 46.9\% indicó haber pensado, visto o escuchado información sobre el COVID-19 entre 1 a 3 horas a la semana, y, la mayor cantidad de las personas no ha recibido diagnóstico y tratamiento previo frente a la ansiedad $(90.7 \%)$. En la Tabla 1 se presenta información detallada sobre las características de las personas. 


\section{Tabla 1}

Descripción de las características de los participantes

\begin{tabular}{|c|c|c|}
\hline Categoría & Frecuencia & $\%$ \\
\hline \multicolumn{3}{|l|}{ Sexo } \\
\hline Hombres & 227 & 63.8 \\
\hline Mujeres & 129 & 36.2 \\
\hline \multicolumn{3}{|l|}{ Estado Civil } \\
\hline Soltero & 333 & 93.5 \\
\hline Casado & 7 & 2.0 \\
\hline Viudo & 1 & 0.3 \\
\hline Conviviente & 15 & 4.2 \\
\hline \multicolumn{3}{|l|}{ Convivencia Familiar } \\
\hline Vive solo & 26 & 7.3 \\
\hline Vive con ambos padres & 181 & 50.8 \\
\hline Vive solo con la madre & 97 & 27.2 \\
\hline Vive solo con el padre & 11 & 3.1 \\
\hline Vive con otros familiares & 41 & 11.5 \\
\hline \multicolumn{3}{|l|}{ Trabajo } \\
\hline Trabajo fijo & 21 & 5.9 \\
\hline Trabajo temporal & 80 & 22.5 \\
\hline Desempleado & 255 & 71.6 \\
\hline \multicolumn{3}{|l|}{ Diagnóstico de Covid - 19} \\
\hline Diagnostico positivo de COVID - 19 & 4 & 1.1 \\
\hline Diagnostico negativo de COVID - 19 & 352 & 98.9 \\
\hline \multicolumn{3}{|l|}{ Diagnóstico COVID - 19 familia } \\
\hline Diagnóstico positivo familiares cercanos & 42 & 11.8 \\
\hline Diagnóstico positivo familiares lejanos & 314 & 88.2 \\
\hline \multicolumn{3}{|l|}{ Diagnóstico COVID - 19 amigos } \\
\hline Diagnóstico positivo amigos & 85 & 23.9 \\
\hline Diagnóstico negativo amigos & 271 & 76.1 \\
\hline \multicolumn{3}{|c|}{ Horas ha pensado, visto o escuchado información sobre COVID - 19} \\
\hline 1 a 3 horas & 167 & 46.9 \\
\hline 3 a 5 horas & 77 & 21.6 \\
\hline 5 a 7 horas & 44 & 12.4 \\
\hline Más de 7 horas & 68 & 19.1 \\
\hline \multicolumn{3}{|l|}{ Diagnóstico y tratamiento contra la ansiedad } \\
\hline $\mathrm{Si}$ & 33 & 9.3 \\
\hline No & 323 & 90.7 \\
\hline
\end{tabular}




\section{Instrumentos}

Ficha sociodemográfica: Los universitarios indicaron su edad, sexo, situación laboral, diagnóstico de COVID-19 familiares, amigos y de ellos mismos, diagnóstico y tratamiento contra la ansiedad, y, tiempo pensando, viendo o escuchando información sobre el COVID-19.

Coronavirus Anxiety Scale (CAS, Lee, 2020). El CAS contiene 5 ítems y fue creada en base a artículos revisados en la literatura psicológica acerca del miedo y la ansiedad, los cuales incluían contenido cognitivo (Pensamiento repetitivo; preocupación; sesgos de procesamiento; soñar; planificar), conductual (Actividades disfuncionales; evitación; conductas compulsivas), emocional (Miedo; ansiedad; enojo) y fisiológico (Trastornos del sueño; angustia somática; inmovilidad tónica;). Se utilizó la versión adaptada y validada en el Perú (Caycho-Rodríguez et al., 2020), donde los participantes calificaban con qué frecuencia experimentaban cada síntoma de ansiedad en una escala tipo Likert de 5 puntos $(0=$ Nada a $4=$ Casi todos los días durante las últimas 2 semanas). Asimismo, la sumatoria de las puntuaciones de cada ítem permite obtener una puntuación total que varía entre 0 y 20 , donde las puntuaciones más altas indican niveles más altos de ansiedad por COVID-19. En el presente estudio, el CAS muestra adecuadas evidencias de fiabilidad $(\alpha=0.86)$.

Mental Health Inventory-5 (MHI-5, Rivera-Riquelme, Piqueras, \& Cuijpers, 2019). Es una adaptación simplificada del Mental Health Inventory de 38 ítems desarrollada para evaluar la salud mental en adultos (McHorney \& Ware, 1995). El inventario contiene 5 ítems que miden de manera particular, el estado de ánimo, la experiencia de bienestar psicológico y la ausencia de angustia en el último mes. Se utilizó la versión en español (Rivera-Riquelme, et al., 2019), que tiene una escala Likert simplificada de 4 puntos $(0=$ nunca, $1=$ a veces, $2=$ varias veces y $3=$ siempre). El puntaje total varía de 0 a 15 , con puntajes más altos que indican una mejor salud mental, mientras que puntajes más bajos indican lo contrario. En la presente investigación el MHI muestra adecuadas evidencias de fiabilidad $(\alpha=0.77)$.

\section{Procedimiento}

La investigación se realizó durante la décima y undécima semana del periodo de cuarentena por la pandemia de la COVID - 19 en el Perú (mayo e inicios de junio de 2020). La encuesta (ficha sociodemográfica, el CAS en español y el MHI-5) se administró por Google Forms, lo cual facilitó su alcance y acceso. Asimismo, el enlace de la encuesta se propagó en diversas redes sociales (Facebook, Instagram y Twitter) y por correo electrónico. Durante la encuesta virtual se comunicó el objetivo del estudio, la confidencialidad de los datos, carencia de riesgos y la autonomía de tener la libertad de retirarse de la investigación en el instante que lo crea 
conveniente. La participación fue netamente voluntaria y solo se registraron aquellos que aprobaron el consentimiento informado brindado. Completar la encuesta virtual demoró aproximadamente de 10 a 15 minutos. El protocolo del proyecto de investigación fue aprobado por el Comité de Ética de la Universidad Privada del Norte y siguió los lineamientos de la Declaración de Helsinki.

\section{Análisis de datos}

Primero, se realizó un Análisis Factorial Confirmatorio (AFC) que especificaba dos factores (ansiedad por el COVID-19 y salud mental) para evaluar la relación entre las variables. Se utilizó el estimador de mínimos cuadrados ponderados diagonalmente con media y varianza corregida (WLSMV) debido a la naturaleza ordinal de los ítems (Brown, 2015). La estimación del ajuste para el AFC se basó en la prueba de chi-cuadrado $(\chi 2)$, el error cuadrático medio de aproximación (RMSEA), la raíz cuadrática media estandarizada residual (SRMR), y el índice de ajuste comparativo (CFI). Los valores de ajuste considerados aceptables fueron CFI > .90, RMSEA, SRMR <.08 (DiStefano, Liu, Jiang, \& Shi, 2018; Kline, 2015; Schumacker \& Lomax, 2015).

Para realizar las comparaciones de los datos, se aplicó la $t$ de student para dos muestras independientes y el análisis de varianza de una vía ANOVA para más de 2 grupos independientes. Asimismo, se calculó el tamaño del efecto de las diferencias con la $d$ de Cohen, donde un valor $d=.20$ indica un tamaño del efecto pequeño, $d=.50$ : mediano, y $d=.80$ : grande (Ferguson, 2009). También se calculó el coeficiente omega cuadrado $\left(\Omega^{2}\right)$, donde el valor de .04 reporta un tamaño del efecto mínimo, .25: moderado, y .64: fuerte (Ferguson, 2009). Finalmente, para las correlaciones entre variables se calculó el coeficiente de correlación de Pearson para medir el grado de relación de dos variables cuantitativas y continuas.

Los análisis comparativos se realizaron con SPSS 24.0 para Windows; mientras que el modelo AFC se realizó con el entorno RStudio (RStudio Team, 2018) para R (R Core Team, 2019), utilizando el paquete "lavaan" (Rosseel, 2012).

\section{RESULTADOS}

\section{Análisis correlacional}

Se propuso un modelo de variables latentes para evaluar la relación entre la ansiedad por Covid-19 y la salud mental. El modelo estructural presentó índices de ajuste adecuados $\left(\chi_{(34)}^{2}=50.35, p=.03\right.$, RMSEA $=.04$ [IC90\%: .01 -.06], CFI $=.99$, SRMR $=.06$ ), además que el modelo de medición fue adecuadamente representado por sus ítems. La Figura 1 indica que la ansiedad por el Covid-19 se relaciona negativamente con la salud mental $(\rho=-.67, \mathrm{p}<.01)$ 


\section{Análisis Comparativo}

La tabla 2, muestra las comparaciones de la ansiedad por el covid-19 y la salud mental en función al sexo. Se reportan diferencias estadísticamente significativas y tamaño del efecto pequeño de la ansiedad por COVID - 19 a favor de las mujeres. Asimismo, los hombres reportaron una mayor salud mental que las mujeres, siendo esta diferencia estadísticamente significativa y con un tamaño del efecto pequeño.

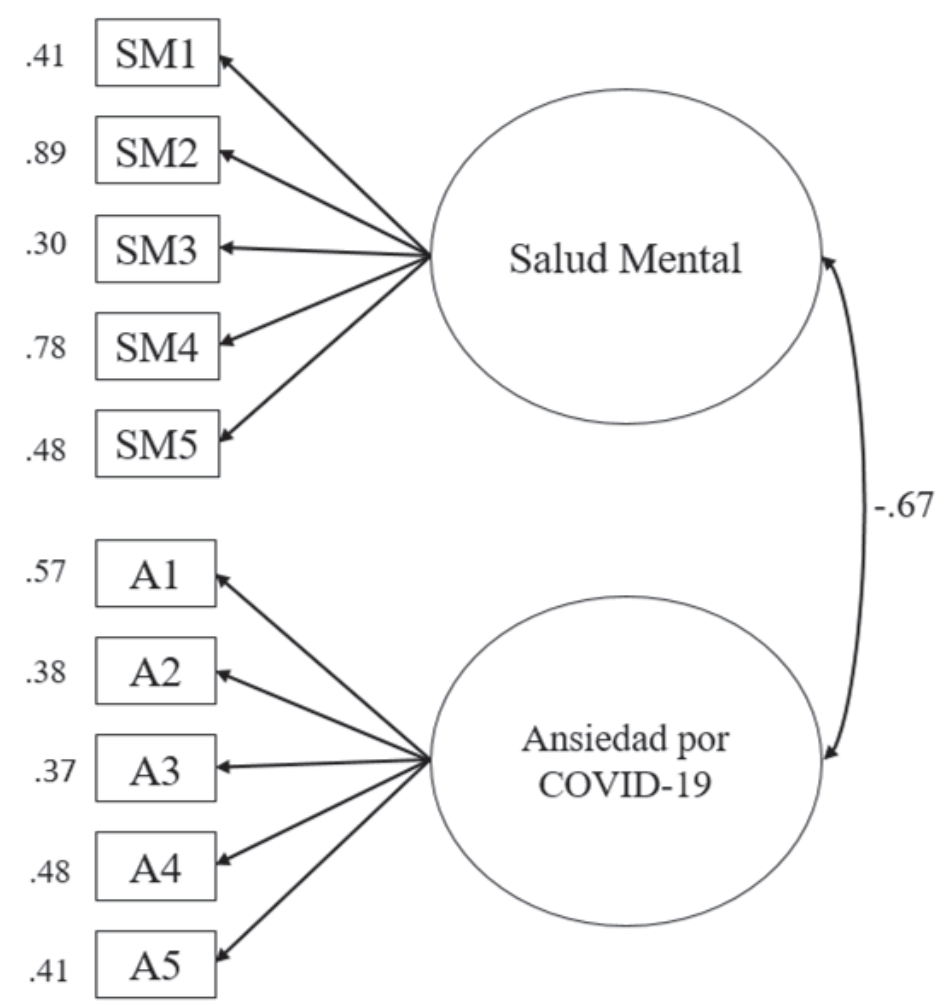

Figura 1. Modelo de relación entre constructos salud mental y ansiedad por el COVID-19

Tabla 2

Comparación de la ansiedad por el COVID-19 y salud mental según el sexo

\begin{tabular}{lccccc}
\hline & Hombres & Mujeres & t & sig & d (IC955\%) \\
& Media (DE) & Media (DE) & & & \\
\hline Ansiedad & $2.77(3.60)$ & $4.11(3.85)$ & 3.25 & 0.001 & $0.36(0.145-0.581)$ \\
Salud Mental & $9.71(2.93)$ & $8.54(2.74)$ & -3.77 & 0.000 & $0.41(0.191--0.64)$ \\
\hline
\end{tabular}


Respecto a la comparación de la ansiedad por el COVID - 19 y la salud mental según el estatus laboral, la tabla 3 indica que los universitarios que se encuentran desempleados presentan mayor ansiedad que los demás grupos, siendo esta diferencia estadísticamente significativa, pero sin tamaño del efecto. Por otro lado, se reporta un tamaño de efecto moderado al comparar la salud mental según se tenga o no trabajo.

Tabla 3

Comparación de la ansiedad por el COVID-19 y salud mental según tipo de trabajo

\begin{tabular}{lllllll}
\hline & $\begin{array}{c}\text { Tengo un trabajo } \\
\text { fijo } \\
\text { Media (DE) }\end{array}$ & $\begin{array}{c}\text { Tengo un trabajo } \\
\text { temporal } \\
\text { Media (DE) }\end{array}$ & $\begin{array}{c}\text { Estoy } \\
\text { desempleado } \\
\text { Media (DE) }\end{array}$ & F & sig & $\mathbf{\Omega}^{\mathbf{2}}$ \\
\hline Ansiedad & $2.57(3.36)$ & $2.95(2.99)$ & $3.93(4.04)$ & 2.86 & 0.058 & 0.01 \\
Salud Mental & $3.14(0.69)$ & $2.47(0.28)$ & $2.94(0.18)$ & 2.21 & 0.111 & 0.39 \\
\hline
\end{tabular}

En la tabla 4, se observa la comparación de la ansiedad por el covid-19 y salud mental según los familiares que han sido diagnosticados con COVID - 19. Se reportan diferencias estadísticamente significativas de la ansiedad por COVID - 19 en los estudiantes universitarios que tienen familiares muy cercanos que han sido diagnosticados con la enfermedad. Asimismo, no existe tamaño del efecto.

\section{Tabla 4}

Comparación de la ansiedad por el COVID-19 y salud mental según diagnóstico de familiares

\begin{tabular}{|c|c|c|c|c|c|}
\hline & $\begin{array}{c}\text { Tener familiares cercanos } \\
\text { diagnosticados con COVID } \\
\text { Media (DE) }\end{array}$ & $\begin{array}{c}\text { Tener familiares lejanos } \\
\text { diagnosticados con COVID } \\
\text { Media (DE) }\end{array}$ & $\mathbf{F}$ & sig & $\mathbf{\Omega}^{2}$ \\
\hline Ansiedad & $5.07(4.16)$ & $3.43(3.73)$ & 6.95 & 0.009 & 0.02 \\
\hline Salud Mental & $8.43(2.78)$ & $9.03(2.73)$ & 1.65 & 0.200 & 0.00 \\
\hline
\end{tabular}

La comparación de la ansiedad por COVID - 19 y salud mental entre aquellos que tienen o no amigos que han sido diagnosticados por COVID - 19 (Tabla 5), indica que los universitarios que tienen amigos diagnosticados con COVID 19 presentan mayor ansiedad por COVID - 19, con un tamaño del efecto pequeño.

Tabla 5

Comparación de la ansiedad por el COVID-19 y salud mental según diagnóstico de amigos

\begin{tabular}{llllll}
\hline & $\begin{array}{c}\text { Tener amigos } \\
\text { diagnosticados con } \\
\text { COVID } \\
\text { Media (DE) }\end{array}$ & $\begin{array}{c}\text { No tener amigos } \\
\text { diagnosticados con } \\
\text { COVID } \\
\text { Media (DE) }\end{array}$ & t & sig & d (IC955\%) \\
\hline Ansiedad & $4.42(4.49)$ & $3.38(3.55)$ & 1.97 & 0.052 & $0.27(0.03-0.519)$ \\
Salud Mental & $8.65(2.42)$ & $9.06(2.99)$ & -1.29 & 0.198 & $0.143(-0.101-0.387)$ \\
\hline
\end{tabular}


Finalmente, en la tabla 6 se observa la comparación de la ansiedad por COVID - 19 y salud mental en función a las horas que han pensado, visto o escuchado información sobre COVID - 19. Se reportó diferencias estadísticamente significativas en los universitarios que pasan más de 7 horas pensando, viendo o escuchando información relacionada al COVID - 19.

Tabla 6

Comparación de la ansiedad por el COVID-19 y salud mental según horas pensando, visto o escuchando información sobre COVID - 19

\begin{tabular}{llllllll}
\hline & $\begin{array}{l}\text { 1 a 3 horas } \\
\text { Media (DE) }\end{array}$ & $\begin{array}{c}\text { 3 a 5 horas } \\
\text { Media (DE) }\end{array}$ & $\begin{array}{c}\text { 5 a 7 horas } \\
\text { Media (DE) }\end{array}$ & $\begin{array}{c}\text { Más de 7 horas } \\
\text { Media (DE) }\end{array}$ & F & sig & $\mathbf{\Omega}^{\mathbf{2}}$ \\
\hline Ansiedad & $3.07(3.52)$ & $3.61(3.85)$ & $4.25(3.94)$ & $4.60(4.20)$ & 3.109 & 0.027 & 0.02 \\
Salud Mental & $9.19(2.91)$ & $9.25(2.57)$ & $8.66(2.92)$ & $8.28(2.96)$ & 2.062 & 0.105 & 0.01 \\
\hline
\end{tabular}

\section{DISCUSIÓN}

El COVID - 19 ha impactado en la salud mental a nivel mundial, generando la aparición de niveles altos de estrés, síntomas de ansiedad, depresión, entre otros, especialmente en grupos de riesgo (Cao et al., 2020; Huarcaya-Victoria, 2020; Ozamiz-Etxebarria, Dosil-Santamaria, Picaza-Gorrochategui, \& Idoiaga-Mondragon, 2020; Parrado-González \& León-Jariego, 2020). Asimismo, un sector fuertemente afectado por la enfermedad de COVID - 19 en el Perú es el educativo, donde alrededor de 1.895 .907 universitarios, entre hombres y mujeres, han visto perjudicados sus estudios (UNESCO, 2020a). En este sentido, en el Perú aún no se ha realizado investigaciones respecto a los problemas de salud mental en el ámbito de la educación superior en el contexto de la pandemia del COVID-19. Por lo tanto, el objetivo de la investigación fue determinar la relación entre la ansiedad por la Covid - 19 y la salud mental. Asimismo, comparar la ansiedad por Covid - 19 y salud mental en función a algunas variables sociodemográficas. Específicamente, se ha reportado una correlación estadísticamente significativa, entre la ansiedad por Covid - 19 y la salud mental en estudiantes universitarios. De la misma manera, se evidenció resultados estadísticamente significativos al comparar la ansiedad por Covid - 19 y salud mental respecto a las variables sociodemográficas: sexo, situación laboral, diagnóstico de Covid -19 en familiares y amigos, y, tiempo pensando, escuchando o viendo noticias sobre la Covid-19. Estos hallazgos se discuten a continuación.

Respecto a la relación entre ansiedad y salud mental, los resultados, coinciden con estudios en China, donde indican que la salud mental de los universitarios se ve afectada de manera significativa al enfrentarse a la pandemia, la cual, ha generado que se posterguen las clases durante meses y se inicie el uso de métodos de aprendizaje a distancia, lo que puede haber ocasionado un impacto negativo 
(Kwok et al, 2020), en diferentes frentes, tales como el pedagógico, socioemocional, financieros y laborales. En relación al ámbito pedagógico, donde se asume que tanto estudiantes como docentes cuentan con el equipo y conectividad necesarios, la educación superior a distancia se concentra en pocas universidades a nivel latinoamericano y el caribe, generando que las competencias de los profesores y estudiantes se vean afectados por el "Coronateaching" que es un síndrome experimentado por el profesor o estudiante al sentirse abrumado por recibir demasiada información por medio de plataformas educativas, aplicaciones, y correo electrónico (Henríquez, 2020; Pedró, 2020; Pérez, 2020). El impacto socioemocional, afecta en mayor medida a los universitarios vulnerables que asistían a programas de nivelación y apoyo, o que tenían problemáticas preexistentes (Pedró, 2020).

En el Perú, no se ha invertido en acceder a campus virtuales, sitios webs institucionales, revistas científicas virtuales, capacitación de docentes y estudiantes en el manejo de las TICS (Tecnologías de la Información y Capacitación), inclusive, le han otorgado poca importancia, lo cual se evidencia en la incapacidad para desarrollar una educación virtual de calidad. Este es un tema poco abordado por algunas universidades públicas dado que ha cuestionado su eficacia. No obstante, muchas universidades privadas peruanas si cuentan con campus virtuales que podrían permitir superar la problemática actual (Ríos, 2020).

Entre los principales estresores académicos se encuentran los retrasos académicos y efectos sobre la vida cotidiana, que se asocian de manera positiva con los síntomas de ansiedad (Cao et al., 2020). Asimismo, los contextos de brotes epidémicos crean diversos desafíos para restaurar la salud al comienzo de la infección y al final de la recuperación; en este proceso, la angustia interna a largo plazo, tanto en el infectado como en la familia del paciente, se ven afectados. Esto puede evidenciarse en diferentes epidemias como la del ébola y la influenza H1N1 donde las personas experimentaron miedo, ansiedad, entumecimiento, desapego, aislamiento, confusión, y conductas de mayor riesgo (fumar, beber, consumo de drogas, etc.) como resultado de la incertidumbre y el confinamiento impuesto por la cuarentena que puede incrementar dichos síntomas o sensaciones afectando su salud mental (OMS, 2014).

Respecto a los resultados comparativos en función al sexo, los hallazgos del presente estudio coinciden con los de Samaniego et. al (2020) quienes reportaron la presencia de sintomatología moderada de estrés (31.92\%), psicosomáticos (5.9\%), dificultades para conciliar el sueño (36.3\%), problemas a nivel social en la actividad diaria (9.5\%) y síntomas de depresión (4.9\%), en especial en las mujeres paraguayas y en los jóvenes (18-25 años). Asimismo, diversas investigaciones reportaron que las mujeres presentaron mayor proporción de síntomas severos y puntajes significativamente más altos que los hombres en ansiedad, y otros estados emocionales negativos debido a la pandemia de COVID - 19 (Orellana \& Orellana, 2020; Sandin, Valiente, Garcia-Escalera \& Chorot, 2020; Valiente, Váz- 
quez, Peinado, Contreras \& Trucharte, 2020; Wang et al., 2020). Esta tendencia puede deberse a la multiplicación de demandas de las labores a nivel doméstico, en relaciones y emociones familiares, reducción de su autonomía y la desatención hacia sí misma por dedicarse a otros, todo ello sumado a sus responsabilidades de trabajo y sus estudios (Comisión Económica para América Latina y el Caribe [CEPAL], 2020; Orellana \& Orellana, 2020; Wenham, Smith \& Morgan, 2020). Sin embargo, la COVID-19 parece ser más contagiosa en hombres, esto genera que la enfermedad sea etiquetada como masculina y se minimice el número de mujeres contagiadas, generando menos atención y cuidados, dejando de lado su salud física y mental (Moreno-Tetlacuilo \& Gutiérrez, 2020). No obstante, se encontraron resultados opuestos reportados por González-Jaimes, Tejeda-Alcántara, Espinosa-Méndez y Ontiveros-Hernández (2020), en donde los universitarios varones mexicanos presentaron puntaciones elevadas de ansiedad (moderada a severa) y las mujeres evidenciaron puntajes más altos (moderados a severos) en síntomas psicosomáticos.

Por otra parte, los resultados al comparar la ansiedad por la COVID - 19 y la salud mental según el estatus laboral son similares a los hallazgos de Orellana y Orellana (2020) quienes reportaron que, las personas que solo estudian y no trabajan, son quienes presentan niveles más altos y generalizados de síntomas emocionales. Los estudiantes presentan varias dificultades en sus deberes, como la improvisada enseñanza y a veces la nula posibilidad para continuar virtualmente con sus estudios, la incertidumbre en relación a los mismos, la separación física de su entorno social y la cancelación de actividades de ocio (Cao et al., 2020; Orellana \& Orellana, 2020; Taylor, 2019). Por otro lado, al comparar la ansiedad por el COVID -19 y salud mental según los familiares que han sido diagnosticados con dicha enfermedad, los resultados son similares a los de Wang, et al. (2020), quienes refieren que tener familia o conocidos con COVID-19 es un factor de riesgo para incrementar la ansiedad de los estudiantes universitarios. Asimismo, otros estudios mencionan que el tener familia o conocidos infectados por Covid - 19, está asociado con un aumento de síntomas de ansiedad por Covid - 19 (Ozamiz-Etxebarria, Dosil-Santamaria, Picaza-Gorrochategui, \& Idoiaga-Mondragon, 2020; Valiente, Vázquez, Peinado, Contreras, \& Trucharte 2020).

Por otro lado, los resultados al comparar la ansiedad por COVID - 19 y salud mental respecto a las horas que han pensado, visto o escuchado información sobre COVID - 19 se asemejan a los de Ozamiz-Etxebarria et al. (2020) quienes manifiestan que en España, la propagación de información errónea acerca de diferentes aspectos sobre COVID - 19 que circulan mediante diferentes medios de comunicación y redes sociales se encuentran al alcance de todo público, especialmente para los jóvenes, lo cual podría explicar su vulnerabilidad psicológica (Orellana \& Orellana, 2020). Asimismo, la sobrecarga de información intensifica los niveles de 
estrés y ansiedad, y puede perjudicar la autoestima (Bawden \& Robinson, 2009; Pantic, 2014; Sevinc \& D’Ambra, 2010; Scholten, et al., 2020).

A pesar de los resultados, el estudio no está libre de limitaciones. Primero, el muestreo fue aplicado por conveniencia, no aleatorio y se ha centrado en estudiantes universitarios de dos ciudades del Perú, ocasionando que no se puedan generalizar los resultados. Se recomienda que en futuras investigaciones se abarquen muestras con mayor alcance a nivel nacional para corroborar los hallazgos. Segundo, se usó un diseño transversal, lo que no permite ver la causalidad entre la ansiedad por COVID - 19 y la salud mental. Es necesario que futuros estudios realicen diseños longitudinales para complementar la información y evaluar las posibles relaciones causales entre ambas variables. Tercero, se utilizaron medidas de autoinforme para obtener los resultados, lo cual, puede generar que se produzcan sesgos de deseabilidad social o de memoria en los participantes. Se sugiere que en investigaciones posteriores utilicen diferentes metodologías, como estudios de casos o entrevistas profundas de índole cualitativo.

Si bien se presentaron limitaciones, también existen fortalezas. En primer lugar, los resultados obtenidos son apropiados para comprender la relación entre la ansiedad por COVID - 19 y la salud mental, y, sus comparaciones con ciertas variables sociodemográficas (sexo, situación laboral, diagnóstico de Covid -19 en familiares y amigos, y, tiempo pensando, viendo o escuchando información sobre el Covid-19). Esto facilitará que futuras investigaciones y profesionales de salud mental puedan identificar grupos vulnerables durante y después de la pandemia de COVID - 19. En segundo lugar, se podrá utilizar la información para fundamentar programas de prevención e intervención para reducir la ansiedad y mejorar la salud mental en los miles de estudiantes universitarios que han sido perjudicados por la pandemia.

\section{CONCLUSIÓN}

En conclusión, los resultados evidenciaron que existe relación entre la ansiedad por el COVID -19 y la salud mental en un grupo de estudiantes universitarios peruanos durante la pandemia. Sin embargo, es imprescindible realizar más estudios a nivel nacional e internacional para complementar los resultados obtenidos, con la finalidad de que surjan intervenciones dirigidas a los estudiantes universitarios que han sido afectados, para mejorar su salud mental.

\section{Fuentes de financiamiento/Funding:}

Recursos propios.

\section{Aspectos éticos/Legales; Ethics/Legals:}

El protocolo del proyecto de investigación fue aprobado por el Comité de Ética de la Universidad Privada del Norte y siguió los lineamientos de la Declaración 
de Helsinki. En la encuesta virtual se comunicó el objetivo de la investigación, la confidencialidad de los datos, carencia de riesgos y la autonomía de retirarse del estudio en cualquier instante. La participación fue netamente voluntaria y solo se registraron aquellos que aceptaron el consentimiento informado.

\section{Conflicto de intereses/Competing interests:}

Los autores manifiestan no tener conflictos de intereses al realizar esta investigación.

\section{REFERENCIAS}

Asmundson, G., \& Taylor, S. (2020a). Coronaphobia: Fear and the 2019-nCoV outbreak. Journal of Anxiety Disorders, 70(1), 1-2. https://doi.org/10.1016/j.janxdis.2020.102196

Asmundson, G., \& Taylor, S. (2020b). How health anxiety influences responses to viral outbreaks like COVID-19: What all decision-makers, health authorities, and health care professionals need to know. Journal of Anxiety Disorders, 71(1). 102211. https:// doi.org/10.1016/j.janxdis.2020.102211

Ato, M., López-García, J. J., \& Benavente, A. (2013). Un sistema de clasificación de los diseños de investigación en psicología. Anales de Psicología, 29(3), 1038-1059.

Bao, Y., Sun, Y., Meng, S., Shi, J., \& Lu, L. (2020). 2019-nCoV epidemic: address mental health care to empower society. Elsevier Public Health Emergency Collection, 395(1), 37-38. https://doi.org/10.1016/S0140-6736(20)30309-3

Bawden, D., \& Robinson, L. (2009). The dark side of information: overload, anxiety and other paradoxes and pathologies. Journal of Information Science, 35(2), 180-191. https://doi.org/10.1177/0165551508095781

Cao, W., Fang, Z., Hou, G., Han, M., Xu, X., Dong J., \& Zheng, J. (2020). The psychological impact of the COVID-19 epidemic on college students in China. Psychiatry Research, 287(1), 1-5. https://doi.org/10.1016/j.psychres.2020.112934

Cardona-Arias, J., Pérez-Restrepo, D., Rivera-Ocampo, S., \& Gómez-Martínez, J. (2015). Prevalencia de ansiedad en estudiantes universitarios. Diversitas Perspectivas en Psicología, 11(1), 79-89. https://doi.org/10.15332/s1794-9998.2015.0001.05

Caycho-Rodríguez, T., Barboza-Palomino, M., Ventura-León, J., Carbajal-León, C., NoéGrijlava, M., Gallegos, M., Reyes-Bossio, M., \& Vivanco-Vidal, A. (2020). Traducción al español y validación de una medida breve de ansiedad por el COVID-19 en estudiantes de ciencias de la salud. Ansiedad y Estrés. En prensa. https://doi. org/10.1016/j.anyes.2020.08.001

Comisión Económica para América Latina y el Caribe (CEPAL). (2020). La pandemia del COVID-19 profundiza la crisis de los cuidados en América Latina y el Caribe. Naciones Unidas: CEPAL Informes Covid-19. https://repositorio.cepal.org/bitstream/ handle/11362/45335/5/S2000261_es.pdf

DiStefano, C., Liu, J., Jiang, N., \& Shi, D. (2018). Examination of the weighted root mean square residual: Evidence for trustworthiness? Structural Equation Modeling: A 
Multidisciplinary Journal, 25(3), 453-466. http://dx.doi.org/10.1080/10705511.2017.1 390394

Ferguson, C. J. (2009). An effect size primer: A guide for clinicians and researchers. Professional Psychology: Research and Pracce, 40(5), 532-538. https://doi.org/10.1037/ a0015808

Fernando, R., \& McKibbin, W. (2020). The Global Macroeconomic Impacts of COVID-19: Seven Scenarios. CAMA Working Paper, 19(2), 1-45. https://doi.org/10.2139/ ssrn.3547729

González-Jaimes, N., Tejeda-Alcántara, A., Espinosa-Méndez, C., \& Ontiveros-Hernández, Z. (2020) Impacto psicológico en estudiantes universitarios mexicanos por confinamiento durante la pandemia por Covid-19. Preprints Scielo. https://doi. org/10.1590/SciELOPreprints.756

Henríquez, F. (16 de abril de 2020). El rol clave del profesor en la educación on line durante la crisis sanitaria. El Universal. https:/eluniversal.cl/contenido/11348/el-rol-clavedel-profesor-en-la-educacion-on-line-durante-la-crisis-sanitaria

Huarcaya-Victoria, J. (2020). Consideraciones sobre la salud mental en la pandemia de COVID-19. Revista Peruana de Medicina Experimental y Salud Publica, 37(2), $327-$ 34. . https://doi.org/10.17843/rpmesp.2020.372.5419

Jaramillo-Valverde, L., \& Marquina, R. (2020). El COVID-19: Cuarentena y su Impacto Psicológico en la población. Preprints Scielo. https://doi.org/10.1590/SciELOPreprints. 452

Johns Hopkins University \& Medicine. (2020). COVID-19 Dashboard by the Center for Systems Science and Engineering (CSSE) at Johns Hopkins University \& Medicine. Baltimore: Coronavirus Resource Center. https://coronavirus.jhu.edu/map.html

Kaplan, H., Sadock, B., \& Sadock, V. (2009). Sinopsis de psiquiatría. 10 ed. Filadelfia: Lippincott Williams \& Wilkins. https://es.slideshare.net/bahulasva/kapaln-ysadock

Kwok, K., Wong, V., Wan In Wei, V., Shan Wong, S., \& Wei-Tze, J. (2020). Novel coronavirus (2019-nCoV) cases in Hong Kong and implications for further spread. Journal of Infection, 80(6), 671-693. https://doi.org/10.1016/j.jinf.2020.02.002

Lai, C., Shih, T., Ko., Tang, H., \& Hsueh, P. (2020). Severe acute respiratory syndrome coronavirus 2 (SARS-CoV-2) and coronavirus disease-2019 (COVID-19): The epidemic and the challenges. International Journal of Antimicrobial Agents, 55(3), 1-9. https://doi.org/10.1016/j.ijantimicag.2020.105924

Lee, S. A. (2020). Coronavirus Anxiety Scale: A brief mental health screener for COVID-19 related anxiety. Death Studies, 44(7), 393-401. https://doi.org/10.1080/07481 187.2020.1748481

Levine, D. \& McKibbin, W. (19 de febrero de 2020). Simple steps to reduce the odds of a global catastrophe. https://www.brookings.edu/opinions/simple-steps-to-reduce-theodds-of-a-global-catastrophe/

Li, S., Wang, Y., Xue, J., Zhao, N., \& Zhu, T. (2020). The Impact of COVID-19 Epidemic Declaration on Psychological Consequences: A Study on Active Weibo Users. Inter- 
national Journal of Environmental Research and Public Health, 17(6), 2032. https:// doi.org/10.3390/ijerph17062032

McHorney, C., \& Ware, J. (1995). Construction and validation of an alternate form general mental health scale for the Medical Outcomes Study Short-Form 36-Item Health Survey. Medical Care, 33(1), 15-28. https://doi.org/10.1097/00005650-199501000-00002

Ministerio de Salud (2020). Sala Situacional COVID-19 Perú. Lima: MINSA. https://covid19.minsa.gob.pe/sala_situacional.asp

Moreno-Tetlacuilo, L. M., \& Gutiérrez, K. (2020). Hombres, mujeres y la COVID-19. ¿Diferencias biológicas, genéricas o ambas? Universidad Nacional Autónoma de México Boletín sobre COVID-19, 1(6), 3-6. http://dsp.facmed.unam.mx/wp-content/ uploads/2013/12/COVID-19-No.6-03-Hombres-mujeres-y-la-COVID-19.pdf

Orellana, C., \& Orellana, L. (2020). Predictores de síntomas emocionales durante la cuarentena domiciliar por pandemia de COVID-19 en El Salvador. Actualidades en Psicología, 34(128), 103-120. https://doi.org/ 10.15517/AP.V34I128.41431

Organización de las Naciones Unidas para la Educación, la Ciencia y la Cultura (UNESCO). (2020a). Education: From disruption to recovery. Unesco Covid-19. https://en.unesco.org/covid19/educationresponse

Organización de las Naciones Unidas para la Educación, la Ciencia y la Cultura(UNESCO). (2020b). Respuesta del ámbito educativo de la UNESCO al COVID-19. Notas temáticas del Sector de Educación. Unesco Covid-19. https://unesdoc.unesco.org/ ark:/48223/pf0000373271_spa?fbclid=IwAR1BBi6CoRKalki-Yi2IpussZMMzzRtHZvIdsyXyPI4D2P92-IKjLWfyOTQ

Organización Mundial de la Salud (OMS). (2014). Primeros auxilios psicológicos para el brote de la enfermedad del virus del Ébola: Informe. Ginebra: Organización Mundial de la Salud. http://www.apps.who.int/iris/bitstream/10665/131682/1/9789241548847_ eng.pdf?ua $=1$

Organización Mundial de la Salud (OMS). (2020). COVID-19: cronología de la actuación de la OMS. Ginebra: Organización Mundial de la Salud. https://www.who.int/ es/news-room/detail/27-04-2020-who-timeline---covid-19

Ozamiz-Etxebarria, N., Dosil-Santamaria, M., Picaza-Gorrochategui, M., \& IdoiagaMondragon, N. (2020). Stress, anxiety, and depression levels in the initial stage of the COVID-19 outbreak in a population sample in the northern Spain. Cadernos de Saúde Pública, 36(4). e00054020. https://doi.org/10.1590/0102-311X00054020

Pantic, I. (2014). Online social networking and mental health. Cyberpsychology, Behavior and Social Networking, 17(10), 652-657. https://doi.org/10.1089/cyber.2014.0070

Parrado-González, A., \& León-Jariego, J. (2020). Covid-19: Factores asociados al malestar emocional y morbilidad psíquica en población española. Revista Española de Salud Pública, 94(8), 1-16. https://www.mscbs.gob.es/biblioPublic/publicaciones/recursos_propios/resp/revista_cdrom/VOL94/ORIGINALES/RS94C_202006058.pdf 
Pedró, F. (2020). Covid-19 y Educación Superior en América Latina y El Caribe: efectos, impactos y recomendaciones políticas. Análisis Carolina, 36(1), 1-15. https://doi. org/10.33960/AC_36.2020

Pérez, C. (10 de abril de 2020). El gran test de las clases online. La Tercera. https://www. latercera.com/tendencias/noticia/el-gran-test-de-las-clases-online/JOJOMO7S2BAB3FNRJYPPHGUZ3I/

Ríos, C. (2020). COVID-19 y Educación Superior Universitaria Pública del Perú. Revista Clake Education, 1(2), 1. http://www.revistaclakeeducation.com/ojs/index.php/Multidisciplinaria/article/view/16/5

Rivera-Riquelme, M., Piqueras, J., \& Cuijpers, P. (2019). The Revised Mental Health Inventory-5 (MHI-5) as an ultra-brief screening measure of bidimensional mental health in children and adolescents. Psychiatry Research, 274(1), 247-253. https://doi. org/10.1016/j.psychres.2019.02.045

Rodríguez, L., Aliaga, J., Peña-Calera, B., \& Quintana, A. (2020). Análisis psicométrico preliminar de la escala perfil de impacto emocional Covid-19 en universitarios peruanos. Revista Educa, 1(15), 1-80.

Rosario-Rodríguez, A., González-Rivera, J. A., Cruz-Santos, A., \& Rodríguez-Ríos, L. (2020). Demandas Tecnológicas, Académicas y Psicológicas en Estudiantes Universitarios durante la Pandemia por COVID-19. Revista Caribeña de Psicología, 4(2), 176-185. https://doi.org/10.37226/rcp.v4i2.4915

Sanchez-Duque, L.A., Arce-Villalobos, L.R., \& Rodríguez-Morales, A.J. (2020). Enfermedad por coronavirus 2019 (COVID-19) en América Latina: Papel de la atención primaria en la preparación y respuesta. Atención Primaria, 52(6), 369-372. https:// dx.doi.org/10.1016\%2Fj.aprim.2020.04.001

Sandin, B., Valiente, R., Garcia-Escalera, J., \& Chorot, P. (2020). Impacto psicológico de la pandemia de COVID-19: Efectos negativos y positivos en población española asociados al periodo de confinamiento nacional. Revista de Psicopatología y Psicología Clínica, 25(1), 1-22. https://doi.org/10.5944/rppc.27569

Sanz, I., Sáinz, J., \& Capilla, A. (2020). Efectos de la crisis del coronavirus en la educación. Organización de Estados Iberoamericanos para la Educación, la Ciencia y la Cultura (OEI). Recuperado de https://oei.org.br/arquivos/informe-COVID-19d.pdf

Scholten, H., Quezada-Scholz, V., Salas, G., Barria-Asenjo, N.A., Rojas-Jara, C., Molina, J., García, J.E., Jorquera, M.T.J., Marinero Heredia, A., Zambrano, A., Gómez Muzzio, E., Cheroni Felitto,A., Caycho-Rodríguez, T., Reyes-Gallardo, T., Pinochet Mendoza, N., Binde,P.J., Uribe Muñoz, J.E., Estupiñan, J.A. \& Somarriva, F. (2020). Abordaje psicológico del COVID-19: una revisión narrativa de la experiencia latinoamericana. Revista Interamericana de Psicología, 54(1), e1287. https://doi. org/10.30849/ripijp.v54i1.1287

Sevinc, G., \& D'Ambra, J. (2010). The influence of self-esteem and locus of control on perceived email overload. Pretoria: 18th European Conference on Information Systems, ECIS 2010. https://www.researchgate.net/publication/221407187_The_Influence_of_Self-Esteem_and_Locus_of_Control_on_Perceived_Email_Overload 
Shigemura, J., Ursano, R., Morganstein, J., Kurosawa, M., \& Benedek, D. (2020). Public responses to the novel 2019 coronavirus (2019-nCoV) in Japan: Mental health consequences and target populations. Psychiatry and Clinical Neurosciences, 74(4). https://doi.org/10.1111/pcn.12988

Soper, D.S. (2020). A-priori Sample Size Calculator for Structural Equation Models. Free Statistics Calculators. http://www.danielsoper.com/statcalc.

Spielberger, C. D., Gorsuch, R. L., Lushene, R., Vagg, P. R., \& Jacobs, G. A. (1983). Manual for the StateTrait Anxiety Inventory. $1^{\mathrm{a}}$ ed. Palo Alto: Consulting Psychologists Press.

Taylor, S. (2019). The psychology of pandemics: Preparing for the next global outbreak of infectious disease. U.K.: Cambridge Scholars Publishing, Newcastle Upon Tyne.

Urzúa, A., Vera-Villarroel, P., Caqueo.Urízar, A., \& Polanco-Carrasco, R. (2020). La Psicología en la prevención y manejo del COVID-19. Aportes desde la evidencia inicial. Terapia Psicológica, 38(1), 103-118. https://doi.org/10.4067/S071848082020000100103

Valiente, C., Vázquez, C., Peinado, V. Contreras, A., \& Trucharte, A. (2020). Síntomas de ansiedad, depresión y estrés postraumático ante el COVID-19: prevalencia y predictores. Vida-Covid-19 Informe Técnico: Universidad Complutense de Madrid. https:// tribuna.ucm.es/revcul/tribunacomplutense/doc24997.pdf

Wang, C., Pan, R., Wan, X., Tan, Y., Xu, L., Ho, C., \& Ho, R. (2020). Immediate Psychological Responses and Associated Factors during the Initial Stage of the 2019 Coronavirus Disease (COVID-19) Epidemic among the General Population in China. International journal of Environmental Research and Public Health, 17(5), 1-25. https:// doi.org/10.3390/ijerph17051729

Wang, C., \& Zhao, H. (2020). The Impact of COVID-19 on Anxiety in Chinese University Students. Frontiers in Psychology, 11(1168). https://doi.org/10.3389/fpsyg.2020.01168

Wenham, C., Smith, J., \& Morgan, R. (2020). COVID-19: The gendered impacts of the outbreak. The Lancet, 395(1). 846-848. https://doi.org/10.1016/S0140-6736(20)30526-2 
